\title{
Geographic Distribution of Isophya pienensis in the Romanian Carpathians (Insecta: Orthoptera: Tettigoniidae)
}

\author{
Ionuţ Ştefan IORGU*, Elena Iulia IORGU
}

\begin{abstract}
"Grigore Antipa" National Museum of Natural History, 1 Kiseleff Blvd, 011341 Bucharest, Romania. *corresponding author, e-mail: ionut.iorgu@antipa.ro
\end{abstract}

Received: October 27,2017; Accepted: November 24, 2017; Available online: December 28, 2017; Printed: December 31, 2017

Abstract. Isophya pienensis Mařan, 1952 is a mesophytic bush-cricket occurring in Romania, western Ukraine, southeastern Poland, Slovakia, northeastern Hungary and a few isolated localities in the Czech Republic and Austria (Chobanov et al., 2016). Species distribution in the Romanian Eastern Carpathians and Apuseni Mountains is reviewed, based on literature and personal data.

Key words: Isophya pienensis, distribution, Romanian Carpathians.

One of the richest in species phaneropterinae genera, Isophya Brunner von Wattenwyl is the most problematic taxa regarding its species-level taxonomy (Heller et al., 2004; Chobanov et al., 2013): the lacking of internal sclerotized male genital organs makes the identification of sympatric Isophya species difficult, yet not impossible when studying the diverse rhythmic structures of male song and their species-specific acoustic mating recognition system (Orci et al., 2005; Orci, 2007; Orci et al., 2010; Heller et al., 2015; Iorgu et al., 2017).

Isophya pienensis inhabits montane meadows with herbaceous vegetation, shrublands with Rubus idaeus and forest clearings and edges. With a severely fragmented population, the species is listed as Near Threatened (NT) in the IUCN Red List, the known area of occupancy (AOO) being estimated to be between 300-1.500 sqkm (Chobanov et al., 2016).

In Romania, the bush-cricket Isophya pienensis occurs in the central and northern areas of Eastern Carpathians and in a small region in Apuseni Mountains (Iorgu, 2012). During recent field surveys conducted in the Romanian Carpathians, I. pienensis was recorded in 38 localities, most of these previously unknown (Fig. 1). These findings highlight the need for further surveys in Romania, completing the gaps in orthopterans geographic distribution.

Material. Personal data: Breaza, $47.64^{\circ} \mathrm{N} 25.31^{\circ} \mathrm{E}, 2011.06 .27$; Izvoarele Sucevei, $47.76^{\circ} \mathrm{N} 25.21^{\circ} \mathrm{E}, 2011.06 .27$; Borsec pass, $46.96^{\circ} \mathrm{N} 25.49^{\circ} \mathrm{E}, 2011.06 .30$; Bistricioara, $47.05^{\circ} \mathrm{N} 25.94^{\circ} \mathrm{E}, 2011.07 .01$; Bradu, $47.01^{\circ} \mathrm{N} 25.85^{\circ} \mathrm{E}, 2011.07 .01$; Abrud, $46.29^{\circ} \mathrm{N} 23.07^{\circ} \mathrm{E}, 2011.07 .08$; Roșia Montană, $46.31^{\circ} \mathrm{N} 23.15^{\circ} \mathrm{E}, 2011.07 .09$; Baia Mare, $47.68^{\circ} \mathrm{N} 23.58^{\circ} \mathrm{E}, 2012.06 .07$; Dorna Candrenilor, $47.36^{\circ} \mathrm{N} 25.29^{\circ} \mathrm{E}, 2012.06 .15$; Hășmașu Mare, $46.69^{\circ} \mathrm{N} 25.83^{\circ} \mathrm{E}, 2012.07 .12$; Sfânta Ana lake, $46.12^{\circ} \mathrm{N} 25.9^{\circ} \mathrm{E}$, 2012.08.06; Săpânța, $47.98^{\circ} \mathrm{N} 23.72^{\circ} \mathrm{E}, 2013.06 .19$; Farcău Moutains, $47.87^{\circ} \mathrm{N} 24.44^{\circ} \mathrm{E}$, 2013.06.19; Poienile de sub Munte, $47.82^{\circ} \mathrm{N} 24.49^{\circ} \mathrm{E}, 2013.06 .20$; Gutâi Mountains, $47.7^{\circ} \mathrm{N} 23.4^{\circ} \mathrm{E}, 2013.06 .20$; Băile Borșa, $47.7^{\circ} \mathrm{N} 24.71^{\circ} \mathrm{E}, 2014.07 .30$; Praid, $46.57^{\circ} \mathrm{N}$ 25.19 ${ }^{\circ} \mathrm{E}, 23.06 .2015$; Satu Mare, $46.34^{\circ} \mathrm{N} 25.43^{\circ} \mathrm{E}, 2015.06 .23$; Munticelu Mountains, 


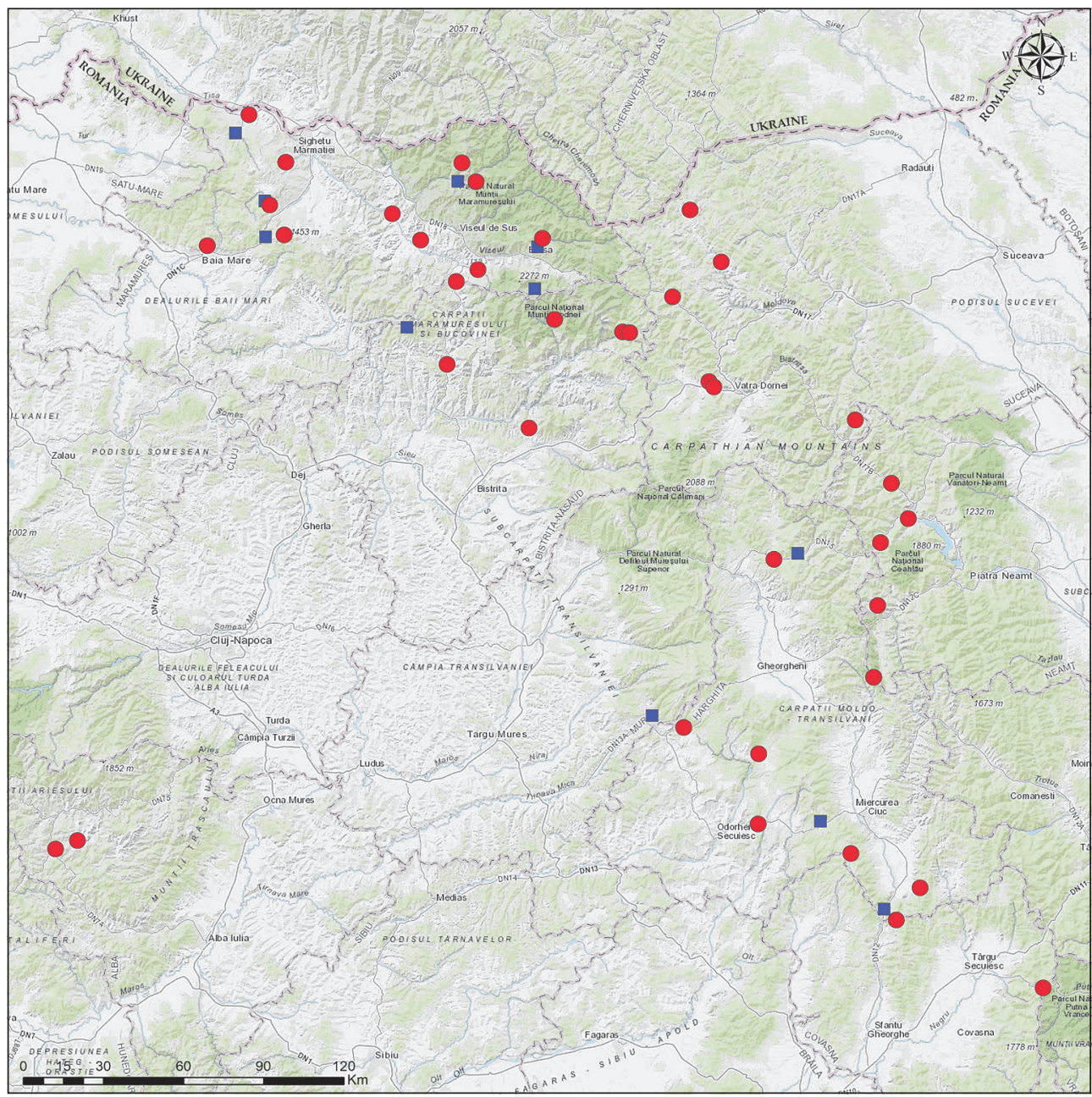

Fig. 1 - Geographic distribution of Isophya pienensis in the Romanian Carpathians. Red circles personal records; blue squares - literature data (other authors, see text).

$46.85^{\circ} \mathrm{N} 25.83^{\circ} \mathrm{E}, 2015.08 .08$; Ilva Mică, $47.26^{\circ} \mathrm{N} 24.67^{\circ} \mathrm{E}, 2017.06 .16$; Șicasău, $46.51^{\circ} \mathrm{N} 25.44^{\circ} \mathrm{E}, 2017.06 .17$; Cașin pass, $46.2^{\circ} \mathrm{N} 25.98^{\circ} \mathrm{E}$, 2017.06.17; Sântimbru Băi, $46.28^{\circ} \mathrm{N} 25.75^{\circ} \mathrm{E}, 2017.06 .19$; Mușat pass, $45.96^{\circ} \mathrm{N} 26.39^{\circ} \mathrm{E}, 2017.06 .19$; Valea Mare, $47.48^{\circ} \mathrm{N} 25.01^{\circ} \mathrm{E}, 2017.06 .24$; Valea Mare, $47.48^{\circ} \mathrm{N} 24.98^{\circ} \mathrm{E}, 2017.06 .24$; Anieș, $47.51^{\circ} \mathrm{N} 24.75^{\circ} \mathrm{E}, 2017.06 .24$; Telciu, $47.41^{\circ} \mathrm{N} 24.39^{\circ} \mathrm{E}, 2017.06 .25$; S Setref pass, $47.6^{\circ} \mathrm{N}$ 24.42 ${ }^{\circ} \mathrm{E}$, 2017.06.25; Săcel, 47.62 ${ }^{\circ} \mathrm{N} 24.49^{\circ} \mathrm{E}, 2017.06 .25$; Bocicoel, $47.69^{\circ} \mathrm{N} 24.3^{\circ} \mathrm{E}$, 2017.06.25; Rozavlea, $47.75^{\circ} \mathrm{N} 24.21^{\circ} \mathrm{E}, 2017.06 .25$; Şugău, $47.87^{\circ} \mathrm{N} 23.85^{\circ} \mathrm{E}, 2017.06 .26$; Mara, $47.77^{\circ} \mathrm{N} 23.79^{\circ} \mathrm{E}, 2017.06 .26$; Dorna Candrenilor, $47.37^{\circ} \mathrm{N} 25.27^{\circ} \mathrm{E}, 2017.07 .05$; Cârlibaba, $47.56^{\circ} \mathrm{N} 25.15^{\circ} \mathrm{E}, 2017.07 .05$; Cotârgași, $47.28^{\circ} \mathrm{N} 25.76^{\circ} \mathrm{E}, 2017.07 .05$; Săvinești, $47.14^{\circ} \mathrm{N} 25.89^{\circ} \mathrm{E}, 2017.07 .05$. 
Literature records (other authors): Baia Borşa (Kis, 1964); Tibleş Mountains, Rodnei Mountains, Sovata, Borsec, Harghita, Tuşnad (Kis \& Vasiliu, 1970); Săpânţa, Mara, Gutâi pass, Poienile de Sub Munte (Szövényi \& Orci, 2008).

\section{ACKNOWLEDGMENTS}

The authors acknowledge T. C. Sahlean for drawing the distribution map and anonymous reviewers for the constructive comments. This study was supported by a grant of the Romanian National Authority for Scientific Research and Innovation, CNCS - UEFISCDI, project number PNII-RU-TE-2014-4-2093.

\section{REFERENCES}

CHOBANOV, D. P., B. GRZYWACZ, I. Ș. IORGU, B. ÇIPLAK, M. B. Ilieva, E. WARCHAŁOWSKAŚLIWA (2013) Review of the Balkan Isophya (Orthoptera: Phaneropteridae) with particular emphasis on the Isophya modesta group and remarks on the systematics of the genus based on morphological and acoustic data. Zootaxa 3658(1): 1-81.

CHOBANOV, D. P., A. HOCHKIRCH, I. S. IORGU, S. IYKOVIC, A. KRIŠTÍN, M. LEMONNIERDARCEMONT, T. PUSHKAR, D. SIRIN, J. SKEJO, G. SZÖVÉNYI, V. VEDENINA, L. P. M. WILLEMSE (2016) Isophya pienensis. The IUCN Red List of Threatened Species 2016: e.T62147304A70269509. Downloaded on 27 October 2017.

HELLER, K.-G., C. HEMP, S. INGRISCH, C. LIU (2015) Acoustic communication in Phaneropterinae (Tettigonioidea) - a global review with some new data. Journal of Orthoptera Research 24(1): $7-18$.

HELLER, K.-G., K. M. ORCI, G. GREIN, S. INGRISCH (2004) The Isophya species of Central and Western Europe (Orthoptera: Tettigonioidea: Phaneropteridae). Tijdschrift voor Entomologie 147: 237-258.

IORGU, I. SS. (2012) First record of Isophya pienensis in the Western Romanian Carpathians (Orthoptera: Phaneropteridae). Brukenthal. Acta Musei VII.3: 481-486.

IORGU, I. Ş., E. I. IORGU, G. SZÖVÉNYI, K. M. ORCI (2017) A new, morphologically cryptic bushcricket discovered on the basis of its song in the Carpathian Mountains (Insecta, Orthoptera, Tettigoniidae). ZooKeys 680: 57-72.

KIS, B. (1964) Contribuţii la cunoaşterea orthopterelor din R.P.R. Studii Universitatea Babeş-Bolyai 2: 69-73. (in Romanian)

KIS, B., M. VASILIU (1970) Kritisches Verzeichnis des Orthopteren-Arten Rumäniens. Travaux du Muséum d'Histoire Naturelle "Grigore Antipa" 10: 207-227. (in German)

ORCI, K. M. (2007) Female preferences for male song characters in the bush-cricket Isophya camptoxypha (Orthoptera, Tettigonioidea). Journal of Insect Behavior 20: 503-513.

ORCI, K. M., B. NAGY, G. SZÖVENYI, I. A. RACZ, Z. VARGA (2005) A comparative study on the song and morphology of Isophya stysi and I. modestior (Orthoptera, Tettigoniidae). Zoologischer Anzeiger 244(1): 31-42.

ORCI, K. M., G. SZÖVÉNYI, B. NAGY (2010) A characterization of the pair forming acoustic signals of Isophya harzi (Orthoptera, Tettigonioidea, Phaneropteridae). Acta Zoologica Academiae Scientiarum Hungaricae 56(1): 43-53.

SZÖVÉNYI, G., K. M. ORCI (2008) Contributions to the Orthoptera fauna of Maramureş, with the first record of Isophya posthumoidalis Bazyluk, 1971 in Romania. Studia Universitatis Vasile Goldiş 18: 235-241. 